\title{
Nutrição enteral com ênfase na composição lipídica: uma revisão
}

\author{
Enteral nutrition with an emphasis on lipid composition: a review \\ Nutrición enteral com enfasis em la composición de lipidos: una revisión
}

\author{
Marciele Alves Bolognese \\ ORCID: https://orcid.org/0000-0003-3417-9566 \\ Universidade Estadual de Maringá, Brasil \\ E-mail: mafb-2006@hotmail.com \\ Patrícia Magalhães de Souza \\ ORCID: https://orcid.org/0000-0001-5916-0744 \\ Universidade Estadual de Maringá, Brasil \\ E-mail: patrícia.magalhaes11@ hotmail.com \\ Vanessa Javera Castanheira Néia \\ ORCID: https://orcid.org/0000-0003-2573-3457 \\ Universidade Estadual de Maringá, Brasil \\ E-mail: nutrivanjavera@ hotmail.com \\ Oscar Oliveira Santos \\ ORCID: https://orcid.org/0000-0002-9631-8480 \\ Universidade Estadual de Maringá, Brasil \\ E-mail: oliveirasantos.oscardeoliveira@ gmail.com \\ Jesuí Vergílio Visentainer \\ ORCID: https://orcid.org/0000-0003-3412-897X \\ Universidade Estadual de Maringá, Brasil \\ E-mail: jesuivv@gmail.com
}

\begin{abstract}
Resumo
A nutrição enteral (NE) fornece nutrientes diretamente ao sistema gastrointestinal para indivíduos que não conseguem deglutir em fases agudas e crônicas. Os lipídios então, assumem papel significativo e são altamente recomendáveis devido ao seu elevado suporte calórico que tem suplementações nas dietas enterais realizadas adicionando-se a estas, alimentos que são fontes ricas em ácidos graxos essenciais, como o, ácidos graxos poli-insaturados (AGPIs), linoleico, e alfa linolênico, visto que ambos AGPIs se associam a inúmeros benefícios a saúde, auxiliando na recuperação rápida dos pacientes. Para o início da terapia nutricional, se faz fundamental um profissional qualificado na área da saúde, que entenda a necessidade do paciente. Logo, o objetivo da presente revisão foi discutir as evidências científicas referentes a composição nutricional lipídica de dietas enterais. A pesquisa foi realizada envolvendo publicações sobre composição lipídica de dietas enterais utilizadas no âmbito domiciliar indexados nas bases de dados eletrônicas PubMed (US National Library of Medicine), Scientific Electronic Library Online Brasil (SciELO), Web of Science e Portal Capes. Os resultados proporcionam aos profissionais de saúde definir tipo de nutrição enteral adequado para o tratamento de seus pacientes, visando a síntese ou manutenção da saúde.

Palavras-chave: Dietas enterais; Nutrição enteral; Lipídios; Ácidos graxos; Ômega 3; Ômega 6; Macronutrientes; Diretrizes.

Abstract

Enteral nutrition (EN) delivers nutrients directly to the gastrointestinal system for individuals who are unable to swallow in acute and chronic phases. Lipids, then, play a significant role and are highly recommended due to their high caloric support that has supplements in enteral diets performed by adding foods that are rich sources of essential fatty acids, such as polyunsaturated fatty acids (PGPIs), linoleic, and alfa linolenic, since both AGPIs are associated with numerous health benefits, helping patients' rapid recovery. For the beginning of nutritional therapy, a qualified professional in the health area, who understands the patient's needs, is essential. Therefore, the aim of this review was to discuss the scientific evidence on the lipid nutritional composition of enteral diets. The research was conducted with publications on the lipid composition of enteral diets used at home, indexed in the electronic databases PubMed (US National Library of Medicine), Scientific Electronic Library Online Brazil (SciELO), Web of Science and Portal Capes. The results allow health professionals to define the type of enteral nutrition suitable for the treatment of their patients, aiming at the synthesis or maintenance of health.
\end{abstract}

Keywords: Enteral diets; Enteral nutrition; Lipids; Fatty acids; Omega 3; Omega 6; Macronutrients; Guidelines.

\section{Resumen}

La nutrición enteral (EN) entrega nutrientes directamente al sistema gastrointestinal para las personas que no pueden tragar en las fases aguda y crónica. Los lípidos, entonces, juegan un papel importante y son muy recomendables 
debido a su alto aporte calórico que tiene suplementos en las dietas enterales que se realizan mediante la adición de alimentos que son fuentes ricas en ácidos grasos esenciales, como los ácidos grasos poliinsaturados (PGPIs), Linoleico, y alfa linolénico, ya que ambos AGPI se asocian con numerosos beneficios para la salud, lo que ayuda a la rápida recuperación de los pacientes. Para el inicio de la terapia nutricional es fundamental un profesional calificado en el área de la salud, que comprenda las necesidades del paciente. Por lo tanto, el objetivo de esta revisión fue discutir la evidencia científica sobre la composición nutricional lipídica de las dietas enterales. La investigación se realizó con publicaciones sobre la composición lipídica de dietas enterales utilizadas en el hogar, indexadas en las bases de datos electrónicas PubMed (Biblioteca Nacional de Medicina de EE. UU.), Scientific Electronic Library Online Brasil (SciELO), Web of Science y Portal Capes. Los resultados permiten a los profesionales de la salud definir el tipo de nutrición enteral adecuada para el tratamiento de sus pacientes, con el objetivo de síntesis o mantenimiento de la salud.

Palabras clave: Dietas enterales; Nutrición enteral; Lípidos; Ácidos grasos; Omega 3; Omega 6; Macronutrientes; Pautas.

\section{Introdução}

A nutrição enteral (NE) é um método que visa o fornecimento de nutrientes diretamente ao sistema trato gastrointestinal, para indivíduos acometidos por doenças em fase aguda ou crônica com dificuldades para deglutir (Perote, Vieira, \& Medeiros, 2014; Garita, Cukier, \& Magnoni, 2009; Waitzber \& Torrinhas, 2016; Kreymann et al., 2006). Geralmente, tais pacientes, apresentam extrema dificuldade em alcançar pelo menos $60 \%$ de suas necessidades nutricionais diárias por via oral, por essa razão a NE é altamente recomendável, auxiliando, sobretudo na manutenção da saúde e/ou recuperação do estado nutricional (Perote, Vieira \& Medeiros, 2014; Garita, Cukier, \& Magnoni, 2009).

A composição das dietas enterais artesanais e industrializadas, devem incluir um equilíbrio de água, eletrólitos (potássio, sódio, cloreto), macronutrientes (proteínas, carboidratos, lipídios), micronutrientes (minerais e vitaminas hidrossolúveis e lipossolúveis), fibras solúveis e insolúveis, bactérias probióticas para compor a flora intestinal do paciente de forma positiva (Muscatiroli et al., 2021). Suas formulações normalmente são baseadas em alimentos frescos, alimentos processados ou alimentos frescos e processados. Portanto, os nutrientes que as compõem são geralmente os mesmos de uma dieta normal, entre eles, carboidratos (40-60\% das necessidades energéticas totais), proteínas (14-20\% das necessidades energéticas totais), fibras e lipídios (15-30\% necessidades energéticas totais) (Gonçalves et al., 2019).

Os lipídios podem ser definidos como um amplo e complexo grupo de compostos orgânicos que geralmente são insolúveis em água, mas solúveis em solventes orgânicos (Damodaran \& Parkin, 2018). Os mesmos desempenham papel extremamente importante para a manutenção da saúde do indivíduo, pois são considerados os componentes principais das membranas, são utilizados como forma de armazenamento de combustível rico de energia na maioria dos organismos e atuam no transporte de vitaminas lipossolúveis (Souza \& Visentainer, 2006). Na nutrição enteral, esse macronutriente assume papel significativo e é amplamente utilizado devido ao seu elevado suporte calórico (variando de 2 a $40 \%$ das calorias totais), sendo também uma ótima opção de fonte concentrada de energia (Calder et al., 2018).

Indivíduos em uso da terapia nutricional enteral precisam de oferta lipídica coerente com sua necessidade, e especializada se for acometido por alguma patologia específica. As suplementações com lipídios nas dietas enterais são realizadas adicionando-se a estas, alimentos que são fontes ricas em ácidos graxos essenciais, em especial, ácidos graxos poliinsaturados (AGPIs) da série ômega 6 (n-6), com ênfase no ácido linoleico (LA, 18:2n-6), e da série ômega-3 (n-3) com ênfase no ácido alfa-linolênico (LNA, 18:3n-3) , como por exemplo, o óleo de soja, emulsões contendo triglicerídeos de cadeia média, azeite de oliva, óleo de peixe entre outros (Calder, 2013; Calder et al., 2018).

O consumo desses AGPIs na nutrição enteral para pacientes em tratamentos, se faz de extrema importância, visto que ambos ácidos graxos n-3 e n-6 associam-se a diversos efeitos benéficos a saúde, como a prevenção e tratamento de enfermidades cardiovasculares, doenças anti-inflamatórias gastrointestinais, infecções, além da prevenção de lesões e 
alterações imunológicas, além de vários outros benefícios (Pradelli, Mayer, Muscaritoli \& Heller, 2012; Andrade \& Carmo, 2006; Calder et al., 2009).

Para o início da terapia nutricional, portanto, se faz fundamental que um profissional qualificado na área, entenda a necessidade do paciente e prescreva uma dieta rica em determinado nutriente a fim de restabelecer a saúde do indivíduo. Nesse âmbito, algumas diretrizes (recomendações publicadas por sociedades de nutrição) estão disponíveis, sendo as mais conhecidas: Sociedade Americana de Nutrição Enteral Parenteral (ASPEN), Sociedade Europeia de Nutrição Clínica e Metabólica (ESPEN) e as Diretrizes Brasileiras em terapia Nutricional (BRASPEN). Nelas, são encontradas recomendações sobre a ingestão de macronutrientes, como proteínas, carboidratos e lipídios, para diferentes tratamentos.

Assim sendo, o objetivo da presente revisão foi realizar uma revisão bibliográfica sobre a composição nutricional lipídica de Nutrição Enteral no âmbito domiciliar.

\section{Metodologia}

O presente trabalho trata-se de um estudo qualitativo de uma revisão narrativa (Pereira, \& Shitsuka, 2018), a qual tem por finalidade discutir, e ampliar o conhecimento sobre a composição nutricional lipídica das dietas enterais no âmbito domiciliar. Essa revisão é constituída por uma análise ampla da literatura, visto que se faz fundamental a aquisição e atualização do conhecimento sobre o tema em foco.

Para o levantamento dos principais e mais relevantes manuscritos relacionados ao tema, foram utilizadas plataformas como Web of Science, Google Scholar, Scopus, PubMed, SciELO e Portal Capes, tendo como período de referência os últimos 10 anos. O critério utilizado para a inclusão das publicações era conter no título, palavras chaves e no resumo, termos vinculados a dietas enterais, dietas domiciliar, nutrição, macronutrientes e composição lipídica (em especial os ácidos graxos da série ômega 3 e ômega 6).

Após escolhido os artigos, foi conduzida, uma leitura minuciosa, destacando os principais tópicos e direcionando uma discussão acerca deste.

\section{Resultados e Discussão}

\subsection{Nutrição Enteral Domiciliar}

A nutrição enteral (NE) é um método que visa o fornecimento de nutrientes diretamente ao sistema trato gastroinstestinal, para indivíduos que não conseguem deglutir em fases agudas e crônicas (Perote, Vieira \& Medeiros, 2014; Garita, Cukier \& Magnoni, 2009; Waitzberg \& Torrinhas, 2016; Kreymann et al., 2006). A NE é indicada para pacientes que apresentam lesões no sistema nervoso central, anorexia, queimaduras, lesões da face e mandíbula, câncer de boca, pancreatite, anormalidades metabólicas do intestino, entre outros (Waitzberg, 2004; Castro \& Cardoso, 2018), Prates \& Anastácia, 2018). Geralmente, tais pacientes, apresentam extrema dificuldade em alcançar pelo menos $70 \%$ de suas necessidades nutricionais diárias por via oral, por essa razão a NE é altamente recomendável, auxiliando sobretudo no desenvolvimento saudável do indivíduo, manutenção da saúde e recuperação do estado/aporte nutricional (Perote, Vieira \& Medeiros, 2014; Garita, Cukier \& Magnoni, 2009).

A RDC n 503 da Agência Nacional de Vigilância Sanitária do Ministério da Saúde, de 2021, estabelece a NE como sendo:

“(...) alimento para fins especiais, com ingestão controlada de nutrientes, na forma isolada ou combinada, de composição definida ou estimada, especialmente formulada e elaborada por uso de sondas ou via oral, industrializada ou não, utilizada exclusiva ou parcialmente para substituir ou complementar a alimentação oral em pacientes 
desnutridos ou não, conforme suas necessidades nutricionais, em regime hospitalar, ambulatorial ou domiciliar, visando a síntese ou manutenção dos tecidos, órgãos ou sistemas."

A alimentação enteral é administrada aos pacientes por meio de um tubo nasogástrico colocado pelo nariz, ou ainda pela gastrostomia (tubo percutâneo colocado no estômago) ou por meio da jejunostomia (tubo percutâneo colocado no intestino delgado). Estudos afirmam que a NE é normalmente considerada segura e o método mais aconselhável para o fornecimento de suporte nutricional, quando comparado por exemplo, a nutrição parenteral (via intravenosa) (Pradelli, Mayer, Muscaritoli \& Heller, 2012).

Comumente a NE é confundida com a alimentação simples. No entanto, deve-se ressaltar que há uma grande diferença entre ambas. A NE está totalmente associada a complicações e riscos, assim ignora totalmente os mecanismos normais de alimentação, como o olfato, paladar, mastigação e deglutição, bem como a fase cefálica da digestão. Além disso, ao contrário dos alimentos normais, as fórmulas entéricas não variam em composição em nutrientes, ou seja, são fixas e definidas de acordo com a necessidade do paciente. Por fim, a NE é fornecida continuamente involuntária ou involuntariamente, em oposição à ingestão intermitente e voluntária de alimento oral (Wanamaker \& Grimm, 2004).

\subsection{Nutrição Enteral artesanal e industrializada}

As dietas enterais podem ser classificadas em artesanais ou industrializadas (Santos, Bottoni, \& Morais, 2013; Zadák \& Kent-Smith, 2009). As dietas enterais industrializadas são as preferíveis e as mais prescritas por médicos e nutricionistas. Apesar de seu custo mais elevado quando comparado as dietas artesanais, elas se destacam por oferecerem maior confiabilidade, segurança (devido ao maior controle sanitário) e uma rotulagem que permite saber exatamente sua composição química e nutricional (Sartori, Rosanelli, Stumm, Kolankiewicz \& Loro, 2013; Jansen et al., 2017).

As dietas artesanais (também conhecidas como não industrializadas ou caseiras), constituem-se de fórmulas elaboradas com alimentos in natura ou produtos alimentícios em geral, que devem ser prescritos por profissionais qualificados, os quais sabem exatamente a composição em macro e micronutrientes necessários para suprir as necessidades de seus pacientes (Mauricio, Gazola \& Matioli, 2008). Essas dietas compõem o que chamamos de nutrição enteral domiciliar (NED). Como o próprio nome sugere, a NED é entregue aos pacientes em estado de vulnerabilidade em suas casas, com a presença de cuidadores. Esse fato é benéfico pois muitas famílias vivem em estado crítico relacionado a fatores econômicos, higiênicosanitários e sociais, e o acesso as dietas industrializadas/ comerciais acaba sendo limitado. Por essa razão as preparações de dietas caseiras são importantes e nesse caso, ideais, visto que auxiliam nos benefícios sociais, melhoria do paciente em acompanhamento domiciliar e diminuição das idas aos hospitais (Mezzomo, Fiori, Reis \& Schieferdecker, 2021).

Apesar da praticidade, esses tipos de dietas apresentam algumas desvantagens, como, estar mais suscetíveis as contaminações microbiológicas, higiene inadequada dos utensílios utilizados, temperatura que favorece o crescimento dos microrganismos, dentre outros (Maniglia, Pagnani \& Nascimento, 2015; Lucas et al., 2018). Felicio, Pinto, Andrade \& Da Silva (2012) realizou um estudo onde foi avaliada a qualidade das dietas enterais artesanais, as quais foram distribuídas em um hospital localizado no vale de Jequitinhonha. Os resultados da pesquisa evidenciaram que tais dietas não supriram as necessidades dos pacientes por ter um baixo suporte calórico. Após, serem realizadas análises físico-químicas nessas mesmas dietas concluíram que houve perda de nutrientes durante a formulação, por essa razão as mesmas acabam não sendo as mais recomendáveis.

\subsection{Classificação das Dietas Enterais}

As dietas enterais podem ser classificadas de diferentes maneiras, sendo elas, quanto a apresentação, indicação, suprimento de calorias, complexidade de nutrientes, presença de elementos específicos, quantidade de proteínas e osmolalidade (Waitzberg \& Torrinhas, 2016; Castro \& Cardoso, Prates, \& Anastácia, 2018; Anvisa; 2015). 
A primeira classificação leva em consideração a apresentação. As NE podem ser encontradas na forma de pó, líquidas semi prontas e líquidas prontas para o uso. No formato de pó, a mesma deve ser reconstituída pela simples adição de água. Já as dietas líquidas semi prontas são reconstituídas industrialmente, e as líquidas prontas, são aquelas que já estão envasadas, prontas para o uso, e mantidas em bolsas ou frascos (Castro, Cardoso, Prates \& Anastácia, 2018; Anvisa, 2015).

A segunda classificação leva em consideração a indicação, sendo ainda divididas em: fórmula padrão, fórmulas modificadas e módulos de nutrientes. Na fórmula padrão a composição em macro e micronutrientes são estabelecidos visando as recomendações para pessoas saudáveis. Já as fórmulas modificadas são aquelas que sofreram alguma alteração em relação aos requisitos de composição estabelecidos pela fórmula padrão para nutrição enteral, essa modificação pode ser na ausência, redução ou aumento de nutrientes, adição de substâncias não previstas ou de proteínas hidrolisadas. Por fim, os módulos de nutrientes são compostos pelos grupos de nutrientes: carboidratos, lipídios, proteínas, fibras alimentares ou micronutrientes (vitaminas e minerais) (Castro, Cardoso, Prates, \& Anastácia, 2018; Anvisa, 2015).

A terceira classificação leva em consideração os suplementos de calorias, sendo eles divididos em: hipocalóricas, normocalóricas e hipercalóricas. As hipocalóricas apresentam densidade calórica inferior a $0,9 \mathrm{kcal} / \mathrm{mL}$, já as normocalóricas tem densidade calórica maior ou igual a $9 \mathrm{kcal} / \mathrm{mL}$ e menor ou igual a 1,2 kcal/mL. E por fim, as hipercalóricas apresentam densidade calórica superior a 1,2 kcal/mL (Castro, Cardoso, Prates \& Anastácia, 2018; Anvisa, 2015).

A quarta classificação leva em consideração a complexidade de nutrientes, sendo as dietas divididas em poliméricas (macronutrientes encontram-se sob sua forma inalterada), oligoméricas/semielementares (macronutrientes encontram-se sob sua forma parcialmente hidrolisada) e hidrolisadas/elementares onde os macronutrientes encontram-se sob sua forma totalmente hidrolisada. A quinta classificação determina sobre a presença de elementos específicos, podendo ser elas: lácteas ou isentas de lactose; com fibras ou isentas de fibras e módulos de nutrientes (Castro, Cardoso, Prates \& Anastácia, 2018; Anvisa, 2015).

Já a quinta classificação leva em consideração a quantidade de proteínas, sendo elas: hipoproteica, onde a quantidade de proteínas é inferior a 10\% do valor energético total, a normoproteica onde a quantidade de proteínas é maior ou igual a 10\% e menor que $20 \%$ do valor energético total, e por fim a hiperproteica sendo a quantidade de proteínas igual ou superior a $20 \%$ do valor energético total (Castro, Cardoso, Prates, \& Anastácia, 2018; Anvisa, 2015).

A última classificação leva em consideração a osmolalidade, neste caso, as dietas são divididas em: hipotônica (280$300 \mathrm{mOsm} / \mathrm{kg}$ de água), isotônica (300-350mOsm/kg de água), levemente hipertônica (350-550mOsm/kg de água), Hipertônica (550-750mOsm/kg de água) e acentuadamente hipertônica (>750mOsm $/ \mathrm{kg}$ de água) (Castro, Cardoso, Prates, \& Anastácia, 2018; Anvisa, 2015).

\subsection{Composição nutricional das dietas enterais}

A composição de nutrição enteral é composta por um equilíbrio de água, eletrólitos (potássio, sódio, cloreto), macronutrientes (proteínas, carboidratos, lipídios), micronutrientes (minerais e vitaminas - hidrossolúveis e lipossolúveis), fibras solúveis e insolúveis, bactérias probioticas para compor a flora intestinal do paciente de forma positiva (Muscatiroli et al., 2021). Suas formulações são baseadas em alimentos frescos, alimentos processados ou alimentos frescos e processados. Portanto, os nutrientes que as compõem são geralmente os mesmos de uma dieta normal, entre eles, carboidratos (40-60\% das necessidades energéticas totais), proteínas (14-20\% das necessidades energéticas totais), lipídios (15-30\% necessidades energéticas totais) e fibras (Gonçalves et al., 2019). Desta forma, a composição contém todos os nutrientes essenciais para uma nutrição equilibrada e saudável, além da intenção de repor componentes do estresse causado por doenças específicas de pacientes atendidos (Coppini, Sampaio, Marco \& Martini, 2011). O manejo de eletrólitos em pacientes deve ser 
individualizado, de acordo com os níveis séricos, a necessidade de reposição depende de resultados séricos de cada paciente e de cada eletrólito (Zambelli et al., 2021).

No geral, o suprimento de micronutrientes como vitaminas e minerais, devem ser redigidos através de uma avaliação nutricional clínica, podendo assim qualquer risco de deficiência de micronutrientes específicos ser corrigido e/ou prevenido imediatamente (Martindale et al., 2020). Existem situações clínicas específicas, quais a avaliação nutricional clínica é indispensável para uso da nutrição enteral ser administrada, por exemplo, casos de insuficiência renal, pois são casos que algumas vitaminas e minerais são insuficientes na dieta, e devem ser indicados suplementação (Fiaccadori et al., 2021; Zambelli et al., 2021).

Em determinação da origem de composição de uma nutrição enteral, relaciona-se sobre os carboidratos, em sua grande maioria estar na forma simples, Singer et al. (2019) menciona que o carboidrato deve ser apresentado em forma de glicose para adultos, originado de frutas de preferência; pode ser justificado também pelo fato da disponibilidade por ingestão a lactose causar intolerância a um certo público, caso preocupante a enfermos adultos. Já sobre a origem lipídica, Gonçalves et al. (2019) mencionam a necessidade de adição em sua formulação origens vegetais nobres, em contrapartida Singer et al. (2009) referem-se à necessidade da emulsão lipídica de ômega-3 (EPA+DHA) na composição.

A adição dos ácidos graxos essenciais ômega-3, ácido eicosapentaenoico (EPA) e do ácido docosaexaenoico (DHA) às emulsões lipídicas de nutrição enteral, apresentam efeitos demonstráveis nas membranas celulares e nos processos inflamatórios (Singer et al., 2009). Autores como Fiaccadori et al. (2021), Sheean et al. (2020), Matsuba et al. (2021) e Singer et al. (2009) relatam que os ácidos graxos ômega-3 são importantes no processo de cicatrização e reposição celular, além de responsáveis por redutores de dores articulares. Para McClave et al. (2016) há carência de evidências sobre alternativas de emulsões lipídicas, porém, faz-se necessidade de suprir os ácidos graxos essenciais.

\subsection{Composição nutricional Lipídica das Dietas Enterais}

Segundo Damodaran e Parkin (2018), os lipídios podem ser definidos como um amplo e complexo grupo de compostos orgânicos que geralmente são insolúveis em água, mas solúveis em solventes orgânicos. Os mesmos são classificados conforme sua polaridade em lipídios neutros e lipídios polares. Os lipídios neutros ou apolares incluem os triglicerídeos, diglicerídeos, monoglicerídeos, esteróis, ceras e ácidos graxos. Já os lipídios polares incluem os fosfolipídios e glicolipídios (Souza \& Visentainer, 2006).

De forma geral, os lipídios desempenham papel extremamente importante para a manutenção da saúde do indivíduo. Eles são considerados os componentes principais das membranas, são utilizados como forma de armazenamento de combustível rico de energia na maioria dos organismos, atuam no transporte de vitaminas lipossolúveis, além de muitas outras funções (Souza \& Visentainer, 2006, 2012).

$\mathrm{Na}$ nutrição enteral, esse macronutriente assume papel significativo e é amplamente utilizado devido ao seu elevado suporte calórico (variando de 2 a 40\% das calorias totais), sendo também uma ótima opção de fonte concentrada de energia (Calder et al., 2018). Os principais ingredientes adicionados a dietas enterais são: óleo de soja, emulsões contendo triacilglicerois de cadeia média (TCM), azeite de oliva e óleo de peixe, visto que cada um deste apresenta composição rica em ácidos graxos essenciais (Calder, 2013; Calder et al., 2018).

\subsection{1 Ácidos graxos}

Os ácidos graxos podem ser definidos como ácidos carboxílicos e representados pela fórmula genérica RCOOH, sendo que o grupamento $\mathrm{R}$ indica um grupamento de cadeia carbônica curta, média ou longa, e diferentes grupos $\mathrm{R}$ também fornecem propriedades físico-químicas distintas as moléculas de ácidos graxos. Os AGs podem ainda ser divididos em ácidos 
graxos saturados (AGS) e insaturados (AGIs), dentro dos AGIs tem-se os monoinsaturados (AGMIs) e os poli-insaturados (AGPIs) (Hewavitharana, Perera, Navaratne, \& Wickramasinghe, 2020; Perini et al., 2010).

Um dos principais aspectos relevantes do fornecimento de lipídios na nutrição enteral, é a oferta adequada de ácidos graxos. As dietas enterais costumam ter uma composição significativa de AGPIs, em especial da série ômega 6 (n-6), com ênfase no ácido linoleico (LA, 18:2n-6), e da série ômega-3 (n-3) com ênfase no ácido alfa-linolênico (LNA, 18:3-3) (Calder, 2015; Calder et al., 2018; Neia et al., 2019). Ambos ácidos graxos são considerados estritamente essenciais, ou seja, são aqueles que não podem ser sintetizados pelo organismo humano, tendo que ser adquiridos por meio da alimentação (Ramalho \& Suarez, 2013).

Os benefícios dos ácidos graxos n-3 são diversos, dentre eles, incluem, alívio de dor, redução nos níveis de triglicerídeos, diminuição da pressão sanguínea em hipertensos e na prevenção de enfermidades cardiovasculares, inibição da proliferação de linfócitos e produção de anticorpos e citocinas (Pradelli, Mayer, Muscaritoli, \& Heller, 2012). Já os ácidos graxos n-6, auxiliam na manutenção da pele e proteção contra feridas e infecções. No entanto, deve-se ter cuidado com a quantidade ingerida deste ácido graxo, visto que seu excesso pode causar distúrbios no organismo de pacientes hospitalizados em estado crítico, e comprometer a evolução do mesmo (Andrade \& Carmo, 2006; Calder et al., 2009).

Ademais, os mesmos são precursores AGs de cadeia longa (AGPI-CL), como o ácido araquidônico (AA, 20:4n-6), o ácido eicosapentaenoico (EPA, 20:5n-3) e o ácido docosahexaenóico (DHA, 22:6n-3) (Barceló-Coblijin, Collison, Jolly, \& Murphy, 2005; Perini et al., 2010).

Os AGPIs-CL são extremamente fundamentais na suplementação nutricional, pois apresentam efeito positivo no estado nutricional de pacientes em risco nutricional. O DHA por exemplo, tem efeito positivo no funcionamento e desenvolvimento da retina e do cérebro. Já o EPA auxilia fortemente na prevenção de doenças inflamatórias e degenerativas, e o AA é essencial para o controle da pressão sanguínea e no controle da agregação plaquetária (Calder, 2015; Neia et al., 2019).

Em diversos países são estipulados valores distintos de razão n-6/n-3, como exemplo, no Canadá o valor recomendado é de 4:1 e 10:1, na França estipula-se um valor de 5:1 e já no Japão há uma variação de 2:1 à 4:1. Organizações como a Food and Agriculture Organization (FAO) e World Health Organization (WHO) recomenda um valor de 5:1 e 10:1. Dentre as razões relatadas, a mais adequada é a razão n-6/n-3 10:1. Essas discrepâncias entre as razões podem ser explicadas devido que nos tempos atuais, ocorreu um desequilíbrio entre a ingestão de ácidos graxos n-6/n-3 por conta da elevada ingestão de alimentos processados e ultra processados e diminuição da ingestão de frutas e verduras, ocasionando em dietas com quantidades inadequadas de ácidos graxos n-3 e, consequentemente, contribuindo para o desenvolvimento de doenças alérgicas, inflamatórias e cardiovasculares (Perini et al., 2010).

\subsection{Comparação das necessidades nutricionais $\mathrm{x}$ oferta de nutrientes das dietas enterais}

Considerando as necessidades nutricionais e de saúde dos pacientes, deve-se iniciar a Terapia Nutricional. Neste âmbito, são consultadas as diretrizes (recomendações publicadas por sociedades de nutrição) para a gestão nutricional de pacientes, como a Sociedade Americana de Nutrição Enteral Parenteral (ASPEN), Sociedade Europeia de Nutrição Clínica e Metabólica (ESPEN) e as Diretrizes Brasileiras em terapia Nutricional (BRASPEN). Nelas, possuem as recomendações de ingestão de macronutrientes, como proteínas, carboidratos e lipídios, para diferentes tratamentos (Tabela 1). 
Research, Society and Development, v. 10, n. 15, e506101523178, 2021

(CC BY 4.0) | ISSN 2525-3409 | DOI: http://dx.doi.org/10.33448/rsd-v10i15.23178

Tabela 1. Comparação das necessidades nutricionais de ingestão x oferta de macronutrientes das dietas enterais.

\begin{tabular}{|c|c|c|c|c|}
\hline Diretriz & Autores & Journal & Tratamento & Recomendações (macronutrientes) \\
\hline \multirow[t]{5}{*}{ ESPEN } & Muscaritoli et al. (2021) & Clinical Nutrition & Nutrição clínica no câncer & Total de 43 recomendações; \\
\hline & & & & Proteínas: entre 1 - 1,5 g / kg / dia; \\
\hline & & & & Carboidratos: não definido; \\
\hline & & & & Lipídios: não definido; \\
\hline & & & & $\begin{array}{l}\text { Uso suplementação com ácidos graxos N-3 de cadeia longa ou óleo } \\
\text { de peixe }\end{array}$ \\
\hline \multirow[t]{4}{*}{ ESPEN } & Fiaccadori et al. (2021) & Clinical Nutrition & Terapia de reposição renal & Total de 32 recomendações; \\
\hline & & & & Proteínas: 0,3-0,5 g / kg / dia (para adulto); \\
\hline & & & & Carboidratos: não definido; \\
\hline & & & & Lipídios: não definido \\
\hline \multirow[t]{5}{*}{ ESPEN } & Singer et al. (2019) & Clinical Nutrition & Tratamento Intensivo & Total de 57 recomendações; \\
\hline & & & & Proteínas: 1,3 g / kg / dia \\
\hline & & & & Carboidratos: $\leq 5 \mathrm{mg} / \mathrm{kg} / \mathrm{min}$ \\
\hline & & & & Lipídios: $\leq 1,5 \mathrm{~g} / \mathrm{kg} / \mathrm{dia}$ \\
\hline & & & & Uso de ômega 3 na dieta, com moderação \\
\hline ASPEN & Sheean et al. (2020) & $\begin{array}{l}\text { Journal of Parenteral } \\
\text { and Enteral Nutrition }\end{array}$ & $\begin{array}{l}\text { Avaliação da composição corporal } \\
\text { em várias populações clínicas }\end{array}$ & $\begin{array}{l}\text { *Relação de métodos de composição corporal relevantes (como, } \\
\text { absortometria de raio-X de dupla energia, ultrassom, e análise de impedância } \\
\text { bioelétrica) }\end{array}$ \\
\hline \multirow[t]{4}{*}{ ASPEN } & Kumpf et al. (2017) & Journal of Parenteral & Suporte nutricional de pacientes & Total de 7 recomendações \\
\hline & & and Enteral Nutrition & adultos com fístula enterocutânea & Proteína: $1.5-2.0 \mathrm{~g} / \mathrm{kg} / \mathrm{dia}$ \\
\hline & & & & Carboidratos: não definido; \\
\hline & & & & Lipídios: não definido \\
\hline \multirow[t]{4}{*}{ ASPEN } & Mehta et al. (2017) & Journal of Parenteral & Suporte nutricional no paciente & Total de 7 recomendações; \\
\hline & & and Enteral Nutrition & pediátrico em estado crítico & Proteínas: $\geq 1,5 \mathrm{~g} / \mathrm{kg} /$ dia; \\
\hline & & & $>1$ mês $<18$ anos & Carboidratos: não definido; \\
\hline & & & & Lipídios: não definido \\
\hline ASPEN & McClave et al. (2016) & Journal of Parenteral & Suporte nutricional em pacientes & Proteínas: 1,2-2,0 g / kg / dia; \\
\hline & & and Enteral Nutrition & adultos em estado crítico & Carboidratos: não definido; \\
\hline & & & & Lipídios: não definido \\
\hline
\end{tabular}


Research, Society and Development, v. 10, n. 15, e506101523178, 2021

(CC BY 4.0) | ISSN 2525-3409 | DOI: http://dx.doi.org/10.33448/rsd-v10i15.23178

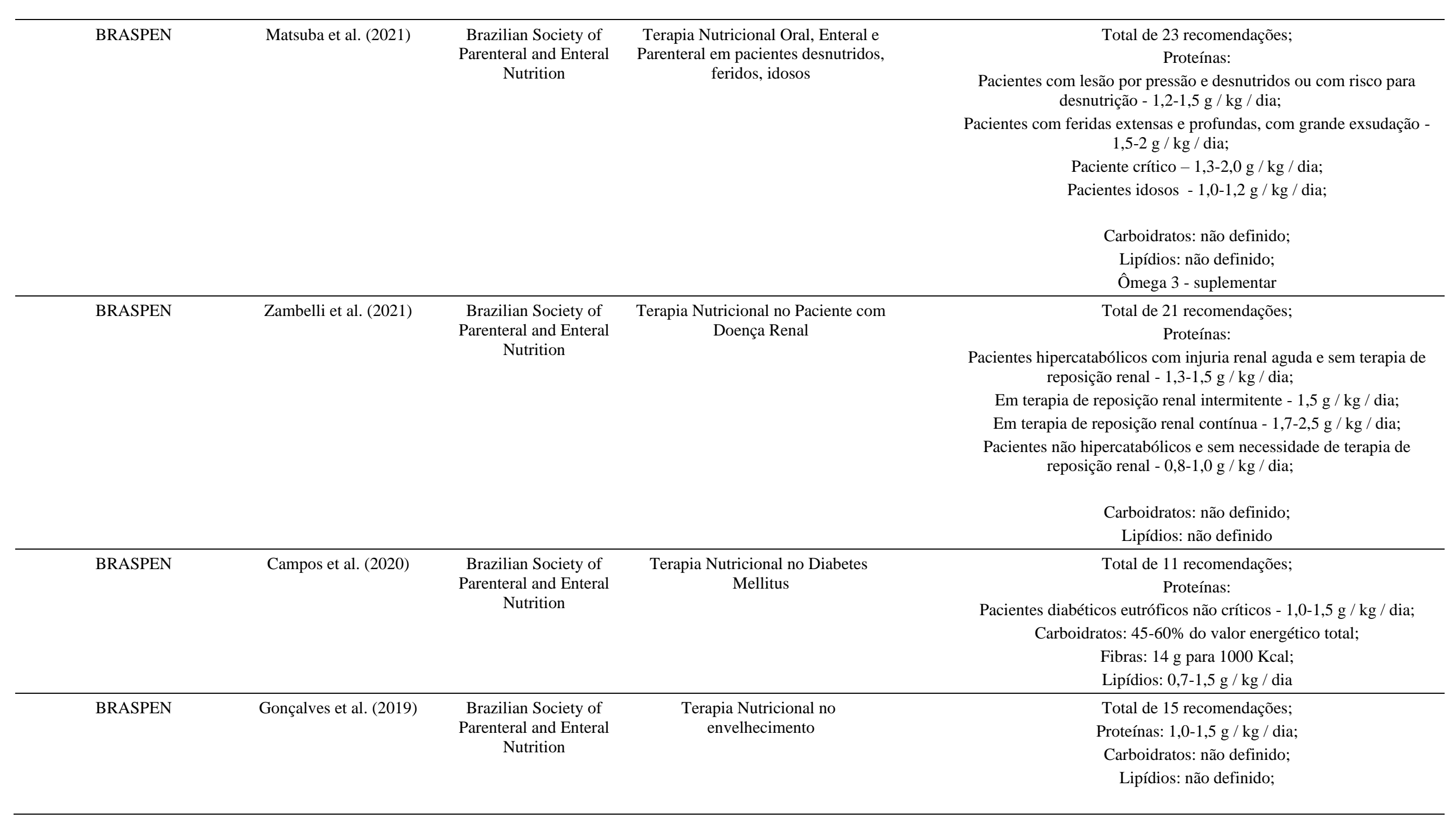




\begin{tabular}{|c|c|c|c|c|}
\hline & & & & Lipídios: $30 \%$ do valor energético total; \\
\hline BRASPEN & Horie et al. (2019) & $\begin{array}{l}\text { Brazilian Society of } \\
\text { Parenteral and Enteral } \\
\text { Nutrition }\end{array}$ & $\begin{array}{l}\text { Terapia nutricional no paciente com } \\
\text { câncer }\end{array}$ & $\begin{array}{l}\text { Total de } 14 \text { recomendações; } \\
\text { Proteínas: } 1,2-2,0 \mathrm{~g} / \mathrm{kg} / \mathrm{dia} \\
\text { Carboidratos: não definido; } \\
\text { Lipídios: não definido }\end{array}$ \\
\hline BRASPEN & Campos et al. (2018) & $\begin{array}{l}\text { Brazilian Society of } \\
\text { Parenteral and Enteral } \\
\text { Nutrition }\end{array}$ & $\begin{array}{c}\text { Paciente grave e Terapia Nutricional } \\
\text { Domiciliar }\end{array}$ & $\begin{array}{l}\text { Proteínas: 1,5-2,0 g / kg/ dia; } \\
\text { Carboidratos: não definido; } \\
\text { Lipídios: não definido }\end{array}$ \\
\hline $\begin{array}{l}\text { Legislação } \\
\text { Brasileira }\end{array}$ & Brasil (2016) & Ministério da Saúde & $\begin{array}{l}\text { Manual de terapia nutricional na } \\
\text { atenção especializada hospitalar }\end{array}$ & $\begin{array}{c}\text { 0 a 12 anos } \\
\text { Proteínas: } 3,0-4,0 \mathrm{~g} / \mathrm{kg} / \mathrm{dia} ; \\
\text { Carboidratos: } 13,0-17,0 \mathrm{~g} / \mathrm{kg} / \mathrm{dia} ; \\
\text { Lipídios: } 3,0-5,0 \mathrm{~g} / \mathrm{kg} / \mathrm{dia} . \\
\text { Adulto e Idosos } \\
\text { Proteínas: } 1,0-4,0 \mathrm{~g} / \mathrm{kg} / \mathrm{dia} ; \\
\text { Carboidratos: Baixo teor de lactose }(<13 \mathrm{dL}) \\
\text { Lipídios: não definido }\end{array}$ \\
\hline $\begin{array}{l}\text { Associação } \\
\text { Médica Brasileira e } \\
\text { Conselho Federal de } \\
\text { Medicina }\end{array}$ & $\begin{array}{l}\text { Coppini, Sampaio, Marco, \& } \\
\text { Martini (2011) }\end{array}$ & $\begin{array}{l}\text { Sociedade Brasileira de } \\
\text { Nutrição Parenteral e } \\
\text { Enteral; Sociedade } \\
\text { Brasileira de Clínica } \\
\text { Médica; Associação } \\
\text { Brasileira de } \\
\text { Nutrologia }\end{array}$ & $\begin{array}{c}\text { Recomendações Nutricionais para } \\
\text { Adultos em Terapia Nutricional } \\
\text { Enteral e Parenteral }\end{array}$ & $\begin{array}{c}\text { Total de } 5 \text { recomendações; } \\
\text { Proteínas: } 1,0-2,0 \mathrm{~g} / \mathrm{kg} / \mathrm{dia} \\
\text { Carboidratos: } \leq 7,0 \mathrm{~g} / \mathrm{kg} / \mathrm{dia} ; \\
\text { Lipídios: entre } 1,0-2,5 \mathrm{~g} / \mathrm{kg} / \mathrm{dia} \\
\text { Ácido graxo linoleico: } 10-17 \mathrm{~g} / \mathrm{dia} \\
\text { Ácido graxo alfa linolenico: } 0,9-1,6 \mathrm{~g} / \mathrm{dia}\end{array}$ \\
\hline $\begin{array}{l}\text { Associação } \\
\text { Médica Brasileira e } \\
\text { Conselho Federal de } \\
\text { Medicina }\end{array}$ & $\begin{array}{l}\text { Coppini, Sampaio, \& } \\
\text { Marco (2011) }\end{array}$ & $\begin{array}{l}\text { Sociedade Brasileira de } \\
\text { Nutrição Parenteral e } \\
\text { Enteral; Sociedade } \\
\text { Brasileira de Clínica } \\
\text { Médica; Associação } \\
\text { Brasileira de } \\
\text { Nutrologia }\end{array}$ & $\begin{array}{c}\text { Recomendações Nutricionais para } \\
\text { Crianças em Terapia Nutricional } \\
\text { Enteral e Parenteral }\end{array}$ & $\begin{array}{c}\text { Proteínas: } \\
\text { Baixo peso ao nascer }-3,0-4,0 \mathrm{~g} / \mathrm{kg} / \mathrm{dia} \\
\text { A termo }-2,0-3,0 \mathrm{~g} / \mathrm{kg} / \mathrm{dia} \\
1 \mathrm{a} 10 \text { anos }-1-1,2 \mathrm{~g} / \mathrm{kg} / \mathrm{dia} \\
10 \mathrm{a} 18 \text { anos (masculino) }-0,9 \mathrm{~g} / \mathrm{kg} / \mathrm{dia} \\
10 \text { a } 18 \text { anos (feminino) }-0,8 \mathrm{~g} / \mathrm{kg} / \mathrm{dia} \\
\text { Em estado grave }-1,5 \mathrm{~g} / \mathrm{kg} / \mathrm{dia}\end{array}$ \\
\hline
\end{tabular}


Research, Society and Development, v. 10, n. 15, e506101523178, 2021

(CC BY 4.0) | ISSN 2525-3409 | DOI: http://dx.doi.org/10.33448/rsd-v10i15.23178

\section{Carboidratos:}

0 a 6 meses $-60 \mathrm{~g} / \mathrm{kg} / \mathrm{dia}$

7 a 12 meses - 95 g / kg / dia;

Demais idades -45 a $65 \%$ do valor energético total;

Lipídios:

0 a 12 meses -30 a $31 \mathrm{~g} / \mathrm{kg} / \mathrm{dia}$;

Demais idades - 25 a $40 \%$ do valor energético total

Fonte: Autores (2021) 
Conforme demonstrado na Tabela 1, foram apresentados 16 estudos e suas recomendações conforme suas Diretrizes específicas. Entretanto, existem limitações sobre as especificações dos teores mínimos e máximos de consumo de macronutrientes em uma dieta qual recorra da nutrição enteral. A partir de estudos e diretrizes estabelecidas, permanece incompreensível em grande maioria uma dieta balanceada para enfermos que necessitam complementar a alimentação com nutrição enteral.

As diretrizes oferecem recomendações básicas que são apoiadas por revisão e análise da literatura atual, além de mencionar outras diretrizes nacionais e internacionais e uma combinação de opinião de especialistas e praticidade clínica (McClave et al., 2016). O uso pretendido dessas diretrizes é para todos os profissionais de saúde envolvidos na terapia nutricional de pessoas em estado crítico - principalmente médicos, enfermeiras, nutricionistas e farmacêuticos.

A realização de atividades físicas em conjunto com a dieta é importante, além disso, em alguns casos faz-se necessário uma maior ingestão de proteínas na dieta quando realizado atividades físicas recorrentes, afim de superar uma resistência anabólica (Singer et al., 2019). Mas o excesso de proteínas pode originar prejuízos nos rins, em especial nas pessoas com insuficiência renal, pois sobrecarregam o seu funcionamento.

Interessante o fato de que não há diferenciação de ingestão de teor proteico entre idades pediátricas $>1$ mês $<18$ anos, Mehta et al. (2017) relata que o balanço de proteína negativo pode resultar em perda de massa magra muscular, que tem sido associada a desfechos desfavoráveis em pacientes críticos, além de, a maior ingestão de proteínas pode estar associada a uma mortalidade mais baixa em 60 dias em crianças sob ventilação mecânica.

\section{Considerações Finais}

A partir do estudo realizado, pode-se destacar informações sobre a composição nutricional das dietas enterais, com foco no perfil lipídico, indicadas para pacientes com diferentes tipos de tratamentos. Os resultados permitem que os profissionais de saúde possam fazer uma escolha da nutrição enteral para o tratamento adequado de seus pacientes, baseados em consultas realizadas nas Diretrizes formuladas por Sociedades de Nutrição Clínica e Metabolismo, responsáveis por definir ou estimar uma composição para o alimento, visando a síntese ou manutenção dos tecidos, órgãos ou sistemas. Por fim, durante a presente pesquisa bibliográfica pode-se concluir que os lipídios, principalmente os ácidos graxos ômega-3 e ômega6, apresentam funções importantes para o desenvolvimento do organismo de pacientes.

No demais, recomendamos que haja novas pesquisas referentes a composição nutricional de dietas enterais, visto que, foi perceptível a carência de dados relacionados ao caráter lipídico e de carboidratos entre as respectivas diretrizes alimentares.

\section{Agradecimentos}

Os autores agradecem o Conselho Nacional de Desenvolvimento Científico e Tecnológico (CNPq) e a Coordenação de Aperfeiçoamento de Pessoal de Nível Superior (CAPES) pelo auxílio financeiro.

\section{Referências}

Andrade, P. D. M. M., \& Carmo, M. G. T. (2006). Ácidos graxos n-3: um link entre eicosanóides, inflamação e imunidade. Revista Mn-Metabólica, 8(3), 135143.

Anvisa - Agência Nacional de Vigilância Sanitária. (2015). Regulamento técnico de fórmulas para nutrição enteral. Resolução da diretoria colegiada - RDC $\mathrm{n}^{\circ}$ 21, de 13 de Maio de 2015. Ministério da Saúde. Diário Oficial da União: Brasília, Brasil. https://bvsms.saude.gov.br/bvs/saudelegis /anvisa/2015/rdc0021_13_05_2015.pdf

Anvisa - Agência Nacional de Vigilância Sanitária. (2021). Requisitos mínimos exigidos para a Terapia de Nutrição Enteral. Resolução da diretoria colegiada - RDC n 503, de 27 de Maio de 2021. Ministério da Saúde. Diário Oficial da União: Brasília, Brasil. https://www.in.gov.br/en/web/dou/-/resolucao-rdc-n503-de-27-de-maio-de-2021-322985331 
Barceló-Coblijn, G., Collison, L. W., Jolly, C. A., \& Murphy, E. J. (2005). Dietary $\alpha$-linolenic acid increases brain but not heart and liver docosahexaenoic acid levels. Lipids, 40(8), 787-798.

Brasil. (2016). Ministério da Saúde. Secretaria de Atenção à Saúde. Departamento de Atenção Especializada e Temática. Manual de terapia nutricional na atenção especializada hospitalar no âmbito do Sistema Único de Saúde - SUS [recurso eletrônico] / Ministério da Saúde, Secretaria de Atenção à Saúde, Departamento de Atenção Especializada e Temática. - Brasília: Ministério da Saúde. https://bvsms.saude.gov.br/bv s/publicacoes/manual_terapia_nutricional_atencao_especializada.pdf

Calder, P. C. (2013). Lipids for intravenous nutrition in hospitalised adult patients: a multiple choice of options. Proceedings of the Nutrition Society, 72(3), $263-276$.

Calder, P. C. (2015). Functional roles of fatty acids and their effects on human health. Journal of parenteral and enteral nutrition, 39, 18S-32S.

Calder, P. C., Adolph, M., Deutz, N. E., Grau, T., Innes, J. K., Klek, S., \& Singer, P. (2018). Lipids in the intensive care unit: Recommendations from the ESPEN Expert Group. Clinical Nutrition, 37(1), 1-18.

Calder, P. C., Albers, R., Antoine, J. M., Blum, S., Bourdet-Sicard, R., Ferns, G. A., \& Zhao, J. (2009). Inflammatory disease processes and interactions with nutrition. British Journal of Nutrition, 101(S1), 1-45.

Campos, A. C. L., Matsuba, C. S. T., Philomene, D. J. v. Nunes, D. S. L., Toledo, D. O., Rocha, E. E. M., Correia, F. G., Ceniccola, G. D., Cunha, H. F. R, ..., \& Loss, S. H. (2018). Diretrizes Brasileira de Terapia Nutricional - Paciente grave e Terapia Nutricional Domiciliar. Brazilian Society of Parenteral and Enteral Nutrition, 1-55. https://f9fcfefb-80c1-466a-835e-5c8f59fe2014.filesusr.com/ugd/a8daef_695255f33d114cdfba48b437486232e7.pdf

Campos, L. F., Hafez, V. C., Barreto, P. A, Gonzalez, M. C., Ceniccola, G. D., Abreu, H. B., Alves, J. T. M., Segadilha, N, L. A. L., Kumbier, M., \& Castro, M. G. (2020). Diretriz BRASPEN de Terapia Nutricional no Diabetes Mellitus. Brazilian Society of Parenteral and Enteral Nutrition, 36, 1-32. https://f9fcfefb-80c1-466a-835e-5c8f59fe2014.filesusr.com/ugd/66b28c_77ee5a91b6d14ade864fe0c091afde8c.pdf

Castro e Cardoso, M. G., Prates, S. M. S., \& Anastácia, L. R. (2018). Fórmulas para nutrição enteral padrão e modificada disponíveis no Brasil: Levantamento e classificação. BRASPEN Journal, 33, 402-417. http://arquivos.braspen.org/journal/out-dez-2018/08formulas.pdf

Coppini, L. Z., Sampaio, H., \& Marco, D. (2011). Recomendações Nutricionais para Crianças em Terapia Nutricional Enteral e Parenteral. Associação Médica Brasileira e Conselho Federal de Medicina. http://www.projetodiretrizes.org.br/9_volume/recomendaco es_nutricionais_para_crianc as_em_terapia_nutricional_enteral_e_parenteral.pdf

Coppini, L. Z., Sampaio, H., Marco, D., \& Martini, C. (2011). Recomendações Nutricionais para Adultos em Terapia Nutricional Enteral e Parenteral. Associação Médica Brasileira e Conselho Federal de Medicina. http://www.projetodiretrizes.org.br/9_volume/recomendacoes_ nutricionais_de_adultos_em_terapia_nutricional_enteral_e_parenteral.pdf

Damodaran, S., \& Parkin, K. L. (2018). Química de alimentos de Fennema. Artmed Editora.

Felicio, B. A., Pinto, R. O. M., Andrade, N., \& Da Silva, D. F. (2012). Food and nutritional safety of hospitalized patients under treatment with enteral nutrition therapy in the Jequitinhonha Valley, Brazil. Nutricion hospitalaria, 27(6), 2122-2129.

Fiaccadori, E., Sabatino, A., Barazzoni, R., Carrero, J. J., Cupisti, A., De Waele, E., \& Cuerda, C. (2021). ESPEN guideline on clinical nutrition in hospitalized patients with acute or chronic kidney disease. Clinical Nutrition, 40, 1644-1668.

Garita, F. S., Cukier, C., \& Magnoni, D. (2009). Indicações e prescrição da terapia nutricional. Matusuba CST, Magnoni D, organizadores. Enfermagem em terapia nutricional. São Paulo: Sarvier, 35-55.

Gonçalves, T. J. M., Horie, L. M., Gonçalves, S. E. A. B., Bacchi, M. K., Bailer, M. C., Barbosa-Silva, T. G., Barrére, A. P. N., Barreto, P. A., Campos, L. F., Campos, G. C., Castro, M. G., Celanos, R. M. G., Deniccola, G. D., ..., \& Dock-Nascimento, D. B. (2019). Diretriz BRASPEN de Terapia Nutricional no envelhecimento. Brazilian Society of Parenteral and Enteral Nutrition, 1-68. https://f9fcfefb-80c1-466a-835e5c8f59fe2014.filesusr.com/ugd/a8daef_13e9ef81b44e4f66be32ec79c4b0fbab.pdf

Hewavitharana, G. G., Perera, D. N., Navaratne, S. B., \& Wickramasinghe, I. (2020). Extraction methods of fat from food samples and preparation of fatty acid methyl esters for gas chromatography: A review. Arabian Journal of Chemistry, 13, 6865-6875.

Horie, L. M., Barrére, A. P. N., Castro, M. G., Alencastro, M. G., Alves, J. T. M. A, Dal Bello, P. P., Braghiroli, M. I., Braz, K. C. C., Cangussú, R., Cardenas, T. C., Carvalho, A. M. B., \& Verotti, C. C. G. (2019). Diretriz BRASPEN de Terapia Nutricional no Paciente com Câncer e BRASPEN recomenda: Indicadores de Qualidade em Terapia Nutricional. Brazilian Society of Parenteral and Enteral Nutrition, 1-46. https:/f9fcfefb-80c1-466a-835e5c8f59fe2014.filesusr.com/ugd/a8daef_19da407c192146e085edf67dc0f85106.pdf

Jansen, A. K., Generoso, S. D. V., Guedes, E. G., Rodrigues, A. M., Miranda, L. A. V. D. O., \& Henriques, G. S. (2017). Desenvolvimento de dietas enterais semiartesanais para idosos em atenção domiciliar e análise da composição de macro e micronutrientes. Revista Brasileira de Geriatria e Gerontologia, 20, $387-397$.

Kreymann, K. G., Berger, M. M., Deutz, N. E., Hiesmayr, M., Jolliet, P., Kazandjiev, G., \& Spies, C. E. S. P. E. N. (2006). ESPEN guidelines on enteral nutrition: intensive care. Clinical nutrition, 25(2), 210-223.

Kumpf, V. J., de Aguilar-Nascimento, J. E., Diaz-Pizarro Graf, J. I., Hall, A. M., McKeever, L., Steiger, E., \& American Society for Parenteral and Enteral Nutrition. (2017). ASPEN-FELANPE clinical guidelines: nutrition support of adult patients with enterocutaneous fistula. Journal of Parenteral and Enteral Nutrition, 41, 104-112.

Lucas, J. L. L., Ribeiro, J. C., Furtado, C. C., \& de Menezes, P. M. G. (2018). Comparação entre Dietas Enterais Artesanais e Industrializadas: Uma Revisão da Literatura. UNILUS Ensino e Pesquisa, 15(38), 5-10. 
Maniglia, F. P., Pagnani, A. C. C., \& Nascimento, G. G. (2015). Desenvolvimento de dieta enteral artesanal com propriedades funcionais. Rev. Bras. Nutr. Clin, 30(1), 66-70.

Martindale, R., Patel, J. J., Taylor, B., Arabi, Y. M., Warren, M., \& McClave, S. A. (2020). Nutrition therapy in critically ill patients with coronavirus disease 2019. Journal of parenteral and enteral nutrition, 44, 1174-1184.

Matsuba, C. S. T., Serpa, L. F., Pereira, S. R. M., Barbosa, J. A. G., Côrrea, A. P. A., Antunes, M. S., Anziliera, F., Araújo, A. S. A., Araújo, S., \& Castro, M, G. (2021) Diretriz BRASPEN de Enfermagem em Terapia Nutricional Oral, Enteral e Parenteral. Brazilian Society of Parenteral and Enteral Nutrition, 36, 171. https://f9fcfefb-80c1-466a-835e-5c8f59fe2014.filesusr.com/ugd/66b28c_8ff5068bd2574851b9d61a73c3d6babf.pdf

Mauricio, A. A., Gazola, S., \& Matioli, G. (2008). Non industrialized enteral diets: microbiological analysis and verification of good preparation practices. Revista de Nutricao-Brazilian Journal of Nutrition, 21(1), 29-37.

McClave, S. A., Taylor, B. E., Martindale, R. G., Warren, M. M., Johnson, D. R., Braunschweig, C., \& Compher, C. (2016). Guidelines for the provision and assessment of nutrition support therapy in the adult critically ill patient: Society of Critical Care Medicine (SCCM) and American Society for Parenteral and Enteral Nutrition (ASPEN). JPEN. Journal of parenteral and enteral nutrition, 40(2), 159-211

Mehta, N., Skillman, H., Irving, S., Coss-Bu, J. A., Vermilyea, S., Farrington, E. A., McKeever, L., Hall, A. M., Goday, P. S., \& Braunschweig, C. (2017) Guidelines for the Provision and Assessment of Nutrition Support Therapy in the Pediatric Critically Ill Patient: Society of Critical Care Medicine and American Society for Parenteral and Enteral Nutrition. JPEN Journal of Parenteral and Enteral Nutrition, 41, 706-742.

Mezzomo, R. T., Stangarlin Fiori, S. L., Oliveira Reis, O. L., Schieferdecker, M. E. M. (2021). Nutritional composition and cost of home-prepared enteral tube feeding. Clinical Nutrition ESPEN, 42, Pages 393-399. https://doi.org/10.1016/j.clnesp.2020.12.016.

Muscaritoli, M., Arends, J., Bachmann, P., Baracos, V., Barthelemy, N., Bertz, H., \& Bischoff, S. C. (2021). ESPEN practical guideline: Clinical Nutrition in cancer. Clinical Nutrition, 40, 2898-2913.

Neia, V. J. C., da Silva dos Santos, P. D., Galuch, M. B., dos Santos Pizzo, J., Ito, A. A. R., Santos, O. O., \& Visentainer, J. V. (2019). Fatty Acid Composition and Lipid Profile of Oral/Enteral Nutrition Supplements Available on the Brazilian Market. European Journal of Lipid Science and Technology, 121(6), 1800495

Pereira, A. S., Shitsuka, D. M., Pereira, F. J., \& Shitsuka, R. (2018). Metodologia da pesquisa científica. [eBook]. Santa Maria. Ed. UAB / NTE / UFSM. Available at https://repositorio.ufsm.br/bitstream/handle/1/15824/Lic_Computacao_Metodologia-Pesquisa-Cientifica.pdf?sequence=1

Perini, J. Â. D. L., Stevanato, F. B., Sargi, S. C., Visentainer, J. E. L., Dalalio, M. M. D. O., Matshushita, M., \& Visentainer, J. V. (2010). Ácidos graxos poliinsaturados n-3 e n-6: metabolismo em mamíferos e resposta imune. Revista de Nutrição, 23, 1075-1086.

Perote, G. M., Vieira, R. Q, \& Medeiros, J. L. (2014). Nutrição enteral e risco de contaminação microbiológica: uma revisão de literatura. Nutrivisa - Revista de Nutrição e Vigilância em Saúde, 1, 23-26. https://www.revistanutrivisa.com.br/wp-content/uploads/2014/11/nutrivisa-vol-1-num-3-e.pdf

Pradelli, L., Mayer, K., Muscaritoli, M., \& Heller, A. R. (2012). n-3 fatty acid-enriched parenteral nutrition regimens in elective surgical and ICU patients: a meta-analysis. Critical Care, 16(5), 1-10.

Ramalho, H. F., \& Suarez, P. A. (2013). A química dos óleos e gorduras e seus processos de extração e refino. Revista Virtual de Química, $5(1), 2-15$.

Santos, V. F. N. D., Bottoni, A., \& Morais, T. B. (2013). Qualidade nutricional e microbiológica de dietas enterais artesanais padronizadas preparadas nas residências de pacientes em terapia nutricional domiciliar. Revista de nutrição, 26, 205-214.

Sartori, T., Rosanelli, C. D. L. S. P., Stumm, E. M., Kolankiewicz, A. C. B., \& Loro, M. M. (2013). Vivências de pacientes em uso de sonda para nutrição enteral. Revista de Pesquisa Cuidado é Fundamental Online, 5(1), 3276-3284.

Sheean, P., Gonzalez, M. C., Prado, C. M., McKeever, L., Hall, A. M., \& Braunschweig, C. A. (2020). American Society for Parenteral and Enteral Nutrition clinical guidelines: the validity of body composition assessment in clinical populations. Journal of Parenteral and Enteral Nutrition, $44,12-43$

Singer, P., Berger, M. M., Van den Berghe, G., Biolo, G., Calder, P., Forbes, A., \& Pichard, C. (2009). ESPEN guidelines on parenteral nutrition: intensive care. Clinical nutrition, 28, 387-400.

Singer, P., Blaser, A. R., Berger, M. M., Alhazzani, W., Calder, P. C., Casaer, M. P., \& Bischoff, S. C. (2019). ESPEN guideline on clinical nutrition in the intensive care unit. Clinical nutrition, 38, 48-79.

Souza, N. E., \& Visentainer, J. V. (2006). Colesterol da mesa ao corpo. São Paulo: Livraria Varela, 85.

Waitzber, D. L., Torrinhas, R. S. (2016). Enteral Feeding. Reference Module in Food Science Encyclopedia of Food and Health. https://doi.org/10.1016/B978-0-12-384947-2.00255-5

Waitzberg, D. L. (2004). Nutrição oral, enteral e parenteral na prática clínica. In Nutrição oral, enteral e parenteral na prática clínica (pp. 1858-1858).

Wanamaker, R., \& Grimm, I. (2004). Encyclopedia of Gastroenterology. Gastroenterology, 127(4), 1274-1275.

Zadák, Z., \& Kent-Smith, L. (2009). Basics in clinical nutrition: Commercially prepared formulas. e-SPEN, the European e-Journal of Clinical Nutrition and Metabolism, 5(4), e212-e215.

Zambelli, C. M. S. F., Gonçalves, R. C., Alves, J. T., Araújo, G. T., Gonçalves, R. C. C., Gusmão, M. H. L., Hordonho, A. A. C., Júnior, F. M. L., Machado, J. C., Nascimento, M. M., \& Martins, C. (2021). Diretriz BRASPEN de Terapia Nutricional no Paciente com Doença Renal. Brazilian Society of Parenteral and Enteral Nutrition, 36, 1-31. https://f9fcfefb-80c1-466a-835e-5c8f59fe2014.filesusr.com/ugd/66b28c_0d8c2c5459c04b9283be89cd2e78c3ee.pdf 